\title{
ERRATUM: “A DECADE OF TIMING AN ACCRETION-POWERED MILLISECOND PULSAR: THE CONTINUING SPIN-DOWN AND ORBITAL EVOLUTION OF SAX J1808.4-3658” (2009, ApJ, 702, 1673)
}

\author{
Jacob M. Hartman ${ }^{1,6}$, Alessandro Patruno $^{2}$, Deepto Chakrabarty ${ }^{3}$, Craig B. Markwardt ${ }^{4,5}$, Edward H. Morgan ${ }^{3}$, \\ Michiel VAN DER KLis ${ }^{2}$, AND Rudy WiJNANDs ${ }^{2}$ \\ ${ }^{1}$ Space Science Division, Naval Research Laboratory, Washington, DC 20375, USA; jacob.hartman@nrl.navy.mil \\ ${ }^{2}$ Astronomical Institute “Anton Pannekoek,” University of Amsterdam, Kruislaan 403, 1098 SJ Amsterdam, The Netherlands; a.patruno@uva.nl, \\ m.b.m.vanderklis@uva.nl,r.a.d.wijnands@uva.nl \\ ${ }^{3}$ Department of Physics and Kavli Institute for Astrophysics and Space Research, Massachusetts Institute of Technology, Cambridge, MA 02139, USA; \\ deepto@space.mit.edu,ehm@space.mit.edu \\ ${ }^{4}$ CRESST/Department of Astronomy, University of Maryland, College Park, MD 20742, USA \\ ${ }^{5}$ Astrophysics Science Division, NASA Goddard Space Flight Center, Greenbelt, MD 20771, USA; craigm@milkyway.gsfc.nasa.gov \\ Received 2012 January 24; published 2012 September 17
}

In the published version of this article, the abstract reported a spin-down rate of $(-5.5 \pm 1.2) \times 10^{-18} \mathrm{~Hz} \mathrm{~s}^{-1}$. It should read $(-5.5 \pm 1.2) \times 10^{-16} \mathrm{~Hz} \mathrm{~s}^{-1}$. The body of the text gives the exponent correctly.

The tenth paragraph of Section 3 reads "As noted in H08, this frequency measurement may be complicated by small systematic differences in the observed pulse frequency between the main outburst and its tail, which were observed during the 1998 and 2002 outbursts." Instead, it should note that these differences were seen during the 2002 and 2005 outbursts; the tail of the 1998 outburst was not observed by RXTE.

\footnotetext{
6 National Research Council Research Associate.
} 